Tetrahedron Letters

journal homepage: www.elsevier.com

\title{
Phosphine-Mediated Addition of 1,2-Dicarbonyls to Diazenes: An Umpolung Approach toward $N$-Acyl Hydrazone Synthesis
}

\author{
Kasey C. Haugen, Kevin X. Rodriguez, Aditi P. Chavannavar, Allen G. Oliver and Brandon L. Ashfeld* \\ Department of Chemistry and Biochemistry, University of Notre Dame, Notre Dame, Indiana 46637, United States \\ *Corresponding author.E-mail address: bashfeld@nd.edu
}

\section{ARTICLE INFO}

Article history:

Received

Received in revised form

Accepted

Available online

Keywords:

Hydrazones

Phosphine

Umpolung

Diazenes

Diaoxaphospholene

\section{ABSTRACT}

Using a Kukhtin-Ramirez-like condensation employing hexamethylphosphorous triamide, the addition of 1,2-dicarbonyls to diazenes affords direct access to N-acyl hydrazones in moderate to excellent yields. The strategic assembly of this ubiquitous functional group proceeds through an umpolung disconnect in which an in situ generated acyl anion equivalent reacts with an electrophilic azodicarboxylate to form a new $\mathrm{C}-\mathrm{N}$ bond. The method presented herein complements traditional condensation approaches toward hydrazone synthesis to access important synthetic intermediates and potentially biologically relevant architectures.

2009 Elsevier Ltd. All rights reserved.
The hydrazone functional group constitutes a ubiquitous and versatile nitrogen-containing carbonyl derivative that is prevalent in biologically active molecules. ${ }^{1}$ In addition to their significance as synthetic intermediates and reagents in their own right, hydrazones have a number of practical applications in both pharmaceutical drug discovery and drug delivery systems. For example, small molecules bearing hydrazone functionality exhibit a wide range of biological and pharmacological properties, including anti-microbial, ${ }^{2}$ anti-inflammatory, ${ }^{3}$ antiplatelet, ${ }^{4}$ anti-malarial, ${ }^{5}$ anti-cancer, ${ }^{6}$ and antifungal ${ }^{7}$ (Figure 1). Additionally, it has been demonstrated that hydrazones have activity against a number of central-nervous system disorders, ${ }^{8}$ and more recently have been used as key elements in linking active small molecules to antibodies in the development of antibody drug conjugates (ADCs). ${ }^{9}$ Given the nucleophilicity of the nitrogens and ambiphilic nature of the carbonyl carbon atom, the reactivity of hydrazones has been exploited in such transformations as the Shapiro, ${ }^{10}$ Bamford-Stevens, ${ }^{11}$ and WolffKishner reactions. ${ }^{12}$ They have also served as intermediates in the synthesis of heterocyclic frameworks, such as pyrrazoles. ${ }^{13}$

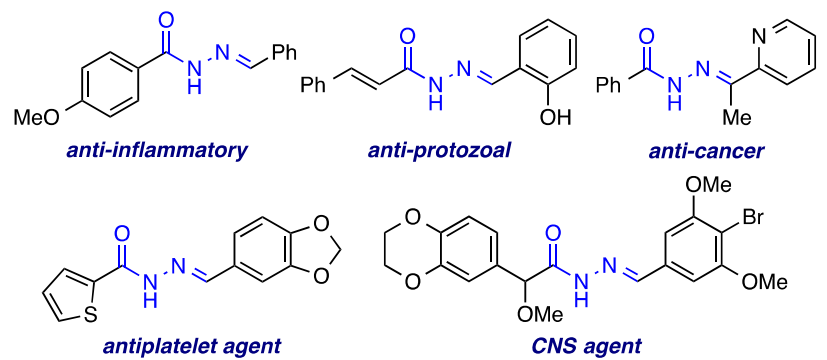

Figure 1. Biologically active $N$-acyl hydrazones.
Conventional methods for synthesizing hydrazones typically involve the condensation of either an electrophilic aldehyde or ketone with hydrazine (Figure 2a). ${ }^{14}$ In contrast, the JappKlingemann reaction, first reported in 1887 by Francis Robert Japp and Felix Klingemann, converts the nucleophilic $\alpha$-carbon of a $\beta$-ketoester to an $N$-aryl hydrazone (Figure 2a). ${ }^{15}$ Enolate addition to an aryl diazonium salt followed by loss of an acetyl group gives the corresponding hydrazone with formal polarity inversion relative to the aforementioned condensation approach. This has proven to be a reliable method to access electron deficient hydrazones bearing a carbonyl derivative at the $\alpha$ position. Unfortunately, inefficient deacetylation of the azo-ester intermediate can occur, and the handling of unstable diazonium salts is often a limitation of this method. ${ }^{16}$ To address these challenges we sought an alternative approach that would enable direct access to electron deficient $N$-acyl hydrazones directly from ketones under neutral conditions. Recently, we reported an umpolung approach to nitrones that relies on the phosphinemediated addition of 1,2-dicarbonyls to nitroso arenes (Figure 2b). ${ }^{17}$ We speculated that a similar approach to hydrazones could be achieved by employing diazenes in place of nitroso arenes. While the formation of hydrazones from diazenes has previously been reported, they are generally few in number and involve either a radical intermediate or the reduction of a prefunctionalized diazene. ${ }^{18}$ Our hypothesis centered on the in situ generation of a stabilized acyl anion equivalent $\mathbf{2}$ by the addition of an electron rich phosphine to ketone $\mathbf{1}$ bearing a resonance anion stabilizing group. Subsequent addition to diazene $\mathbf{3}$ results in formation of a new $\mathrm{C}-\mathrm{N}$ bond en route to hydrazone 4 (Figure 2c). This approach has the advantage of using a nucleophilic carbonyl synthon to facilitate hydrazone formation without resorting to strongly basic conditions. Herein, we report the 
successful development of this umpolung approach toward the synthesis of $N$-acyl hydrazones from 1,2-dicarbonyl substrates.

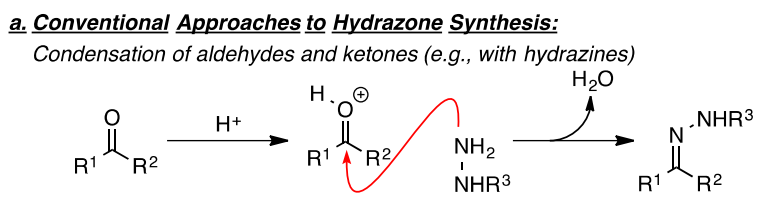

Additions to diazonium salts - the Japp-Klingman reaction

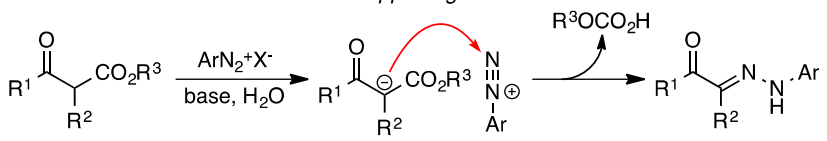

\section{b. Previous work: umpolung addition to nitroso arenes}

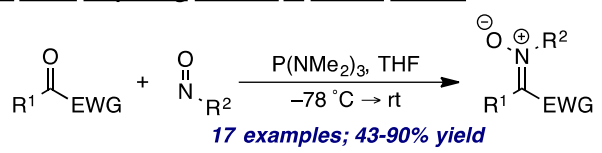

c. This work: polarity inversion = acyl anion equivalent addition to diazenes.

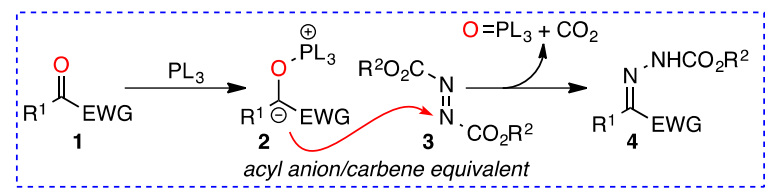

Figure 1. Approaches to hydrazone construction.

We initially evaluated the efficacy of our strategy by examining the phosphine-mediated addition of $\alpha$-ketoester 1a to diisopropylazodicarboxylate (DIAD, 3a, Table 1). Given our success with $\mathrm{P}\left(\mathrm{NMe}_{2}\right)_{3}$ in the addition of 1,2-dicarbonyls to nitroso arenes, we began our study with this phosphorus triamide in slight excess to one equivalent each of $1 \mathbf{a}$ and $3 \mathbf{a}$ at $-78{ }^{\circ} \mathrm{C}$ employing THF as the solvent. Gratifyingly, hydrazone 4a was obtained in $74 \%$ yield after allowing the reaction to warm to room temperature over the course of $1 \mathrm{~h}$ (entry 1 ). We discovered that using a two-fold excess of $\mathbf{1 a}$ improved the yield of $\mathbf{4 a}$ to $84 \%$, but a similar excess of DIAD led to diminished yields (entries 2 and 3). However, employing a 1.2/1.0 ratio of $\mathbf{1 a}$ to $\mathbf{3 a}$ provided $4 \mathbf{a}$ in $95 \%$ yield (entry 4 ). Performing the reaction in PhMe led to results similar to running the reaction in THF, while halogenated and polar aprotic solvents failed to provide hydrazone 4a (entries 5-8). With our optimized conditions in hand, we turned our attention toward evaluating the substrate scope of this method for hydrazone formation.

\begin{tabular}{|c|c|c|c|c|}
\hline Entry & 1a (equiv) & 3a (equiv) & Solvent & Yield (\%) \\
\hline 1 & 10 & 10 & THF & 75 \\
\hline 2 & 2.0 & 1.0 & THF & 84 \\
\hline 3 & 1.0 & 2.0 & THF & 44 \\
\hline 4 & 1.2 & 1.0 & THF & 95 \\
\hline 5 & 1.2 & 1.0 & $\mathrm{PhMe}$ & 94 \\
\hline 6 & 1.2 & 1.0 & $\mathrm{CH}_{2} \mathrm{Cl}_{2}$ & 0 \\
\hline 7 & 1.2 & 1.0 & DMF & 0 \\
\hline 8 & 1.2 & 1.0 & $\mathrm{MeCN}$ & 0 \\
\hline
\end{tabular}

Table 1. Optimization of Reaction Conditions ${ }^{\mathrm{a}}$

Our initial evaluation of functional group tolerance focused on the reaction of $\mathrm{P}\left(\mathrm{NMe}_{2}\right)_{3}$ with various 1,2-dicarbonyls $\mathbf{1}$ and either DIAD (3a) or DEAD (3b) to generate hydrazones 4 (Table 2). In general, application of our optimized conditions led to $\mathrm{N}$ acyl hydrazone formation in good to excellent yields with a variety of $\alpha$-ketoester derivatives. Methyl substitution at the 4and 3-positions of the benzene ring in ketoesters $\mathbf{1 b}$ and $\mathbf{1 c}$ did not adversely effect the reaction outcome in which hydrazones $\mathbf{4 b}$ and $4 \mathbf{c}$ were obtained in $52 \%$ and $67 \%$ yields respectively (entries 1 and 2). It should be noted that in some cases higher yields of hydrazone 4 were obtained employing two equivalents of $\mathbf{1}$, as illustrated by the conversion of $1 \mathbf{c}$ to $\mathbf{4 c}$ in $78 \%$ yield. Benzene-derived aryl ketoesters bearing strongly electron donating groups proceeded in slightly diminished yields in comparison to their electron deficient counterparts. For example, 4-methoxy substituted benzyl formate $1 \mathbf{d}$ gave hydrazone $\mathbf{4 d}$ in $52 \%$ yield, whereas $4-\mathrm{CF}_{3}$-substituted 1 e proceeded in $83 \%$ yield (entries 3 and 4). Likewise, halogen-substituted benzyl formates 1f and $\mathbf{1 g}$ gave the corresponding hydrazones $\mathbf{4 f}$ and $\mathbf{4 g}$ in good yields (entries 5 and 6). Multiple electron withdrawing groups did not hinder the reaction as hydrazone $4 \mathrm{~h}$ was obtained in $54 \%$

Table 2. Evaluation of reaction scope. ${ }^{\mathrm{a}}$

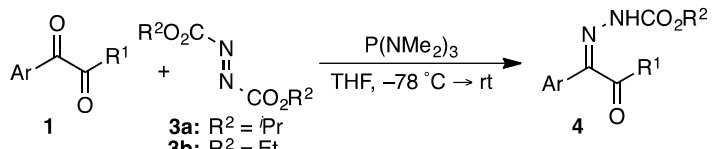

(\%)

${ }^{a}$ Conditions: $1(0.29 \mathrm{mmol})$, 3a or $\mathbf{3 b}(0.24 \mathrm{mmol})$, and $\mathrm{P}\left(\mathrm{NMe}_{2}\right)_{3}(0.37$ $\mathrm{mmol})$ at $-78{ }^{\circ} \mathrm{C}$ to room temperature over $1 \mathrm{~h}$. ${ }^{\mathrm{b}} \mathrm{Tw}$ wo equivalents of $\mathbf{1}$ were employed. 
yield from 3,5-bistrifluoromethyl benzyl 1h (entry 7). Aromatic substituents other than benzene also proved efficacious under the reaction conditions, as exemplified by the conversion of $\mathrm{N}$ methyl imidazole $\alpha$-ketoester $1 \mathbf{i}$ to $4 \mathbf{i}$ in $76 \%$ yield (entry 8 ). In addition to $\alpha$-ketoesters, we discovered that 1,2-diketones were viable substrates in formation of the corresponding hydrazone. Treatment of benzil (1j) with 3a and $\mathrm{P}\left(\mathrm{NMe}_{2}\right)_{3}$ led to formation of hydrazone $\mathbf{4 j}$ in $95 \%$ yield (entry 9). Employing DEAD (3b) in place of DIAD in the conversion of $\alpha$-ketoester 1a led to formation of the corresponding $N$-acyl hydrazone $4 \mathbf{k}$ in comparable yield (entry 10). It deserves noting that $\alpha$-ketoesters bearing alkyl or vinyl substitution resulted in complex mixtures of products, which included primarily formation of the aforementioned epoxide resulting from the formal dimerization of 1.

Diazenes with acyl substitution in place of the more common carboxylates ultimately failed to provide the desired hydrazone. This result is not surprising given the need for a decarboxylation step prior to hydrazone formation. Additionally, employing unsymmetrical diazenes led to a mixture of hydrazone products resulting from an unselective addition of the ketoester to the azodicarboxylate. For example, treatment of mixed diazene $3 \mathbf{c}$ bearing carboxyethyl and carboxybenzyl groups led to an overall $34 \%$ yield of hydrazones $\mathbf{4 k}$ and $\mathbf{4 l}$ in a ratio of 1.3:1.

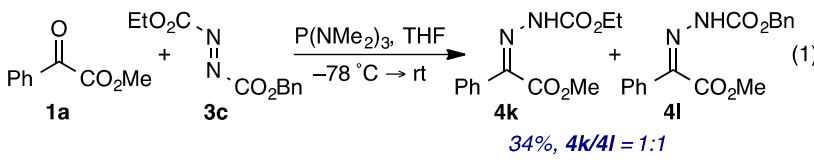

In addition to $\alpha$-ketoesters, we next explored the regiochemistry of hydrazone formation upon subjection of a 1,2,3-tricarbonyl to our optimized reaction conditions. In contrast to our results with unsymmetrical diazenes, treatment of $\alpha, \beta$ diketoester 5 with DIAD (3a) and $\mathrm{P}\left(\mathrm{NMe}_{2}\right)_{3}$ led to clean formation of hydrazone $\mathbf{6}$ wherein regioselective functionalization of the $\alpha$-ketone was the sole product observed (eq. 2). ${ }^{19}$ It should be noted that diketoester 5 was used as a mixture of both the $\alpha$-hydrate and ketone forms to provide $\mathbf{6}$ in $80 \%$ yield. This result would indicate selective addition of phosphine to the $\alpha$-ketone, possibly to avoid steric interactions with the neighboring phenyl ring of the $\beta$-ketone, followed by addition to DIAD.

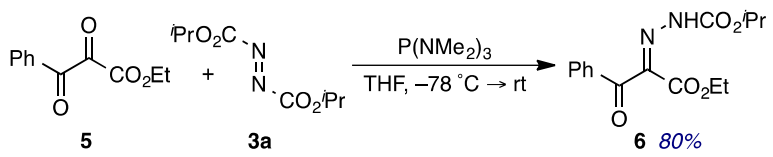

Based on our results, two mechanisms for hydrazone formation are potentially operable from the combination of 1,2dicarbonyls and diazenes. One mechanism involves a KukhtinRamirez-like condensation ${ }^{20}$ in which initial addition of phosphine to the $\alpha$-ketoester yields a zwitterionic complex 2a that is in equilibrium with the corresponding closed hetereocyclic form (Scheme 1a). Based on the pioneering work of Ramirez, this equilibrium likely favors the open, zwitterionic complex depicted given the steric hinderance around the tetravalent phosphorus triamide. ${ }^{21}$ Addition to diazene 3a yields intermediate 7 that leads to a rapid expulsion of phosphine oxide to provide hydrazone precursor 8. Subsequent decarboxylation, possibly via $\beta$-elimination, yields the observed $N$-acyl hydrazone $4 \mathbf{a}$. Alternatively, a Morita-Baylis-Hillman-like mechanism ${ }^{22}$ that involves addition of phosphine to DIAD would lead to hydrazide 9 that then undergoes addition to the electron deficient ketone 1a (Scheme 1b). Phosphoryl transfer converts intermediate $\mathbf{1 0}$ to $\mathbf{1 1}$ through a putative pentavalent phosphorus. Subsequent loss of phosphine oxide gives the common diacyl hydrazone 8 that then undergoes decarboxylation to provide the observed product.

While both mechanisms are conceivable under the reaction conditions, a few key observations are enlightening. First, the rapid formation of epoxide from $\alpha$-ketoesters and $\mathrm{P}\left(\mathrm{NMe}_{2}\right)_{3}$ at low temperatures $\left(-20{ }^{\circ} \mathrm{C}\right)$ would indicate a rapid addition of phosphine to the 1,2-dicarbonyl. Additionally, if the MoritaBaylis-Hillman-like mechanism is operable, one would expect most electrophilic carbonyls to undergo reaction with hydrazide 9. However, exposure of benzaldehyde to our optimized reaction conditions with DIAD (3a) and $\mathrm{P}\left(\mathrm{NMe}_{2}\right)_{3}$ led to no discernable product formation. It would appear that an anion stabilizing electron withdrawing group adjacent to the carbonyl where hydrazone formation occurs is necessary, and is consistent with phosphorane addition to the starting diazene via a KukhtinRamirez-like mechanism.

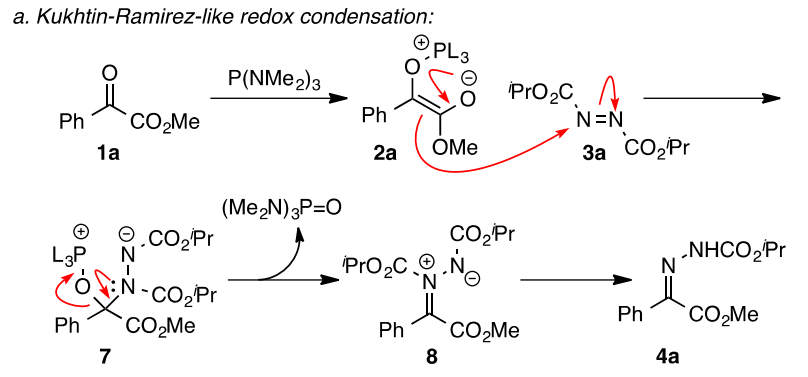

b. Morita-Baylis-Hillman-like addition of hydrazide:
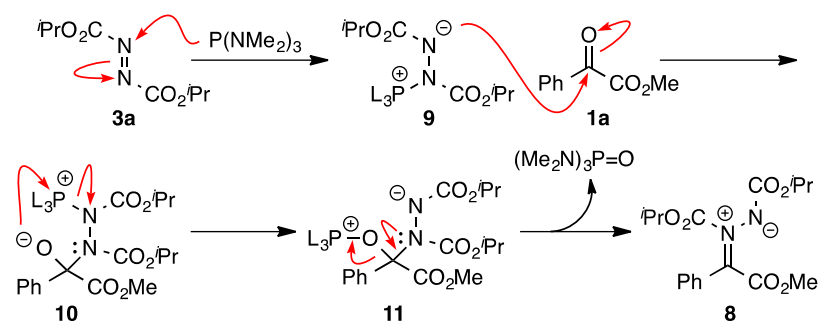

Scheme 1. Possible mechanisms for hydrazone formation.

In conclusion, we have a developed an unconventional approach to $N$-acyl hydrazone synthesis by exploiting a phosphine-mediated Kukhtin-Ramirez-like redox condensation of a 1,2-dicarbonyl and diazene. This method avoids the use of basic amines and highly reactive diazonium salts while employing readily available, easy to handle reagents. The reaction works well with a range of aryl $\alpha$-ketoesters to give the corresponding hydrazones in good to excellent yields. Additionally, 1,2-diketones and $\alpha, \beta$-diketoesters were also shown to be effective substrates in selective hydrazone formation. Additional mechanistic studies are currently underway and will be reported in due course.

\section{Dedication}

This manuscript is dedicated to the memory of Professor Harry Wasserman, an inspirational leader and scholar.

\section{Acknowledgments}

Financial support for this work was provided by the Stanford University Global Climate and Energy Project and the National Science Foundation (CAREER CHE-1056242). 


\section{Supplementary Material}

Supplementary material associated with this article including experimental procedures and compound characterizations can be found, in the online version, at

\section{References and notes}

1. Rollas, S.; Küçükgüzel, Ş. G., Molecules 2007, 12, 1910.

2. (a) Telvekar, V. N.; Belubbi, A.; Bairwa, V. K.; Satardekar, K., Bioorg. Med. Chem. Lett. 2012, 22, 2343. (b) Özkay, Y.; Tunalı, Y.; Karaca, H.; Işı1kdağ, İ., Eur. J. Med. Chem. 2010, 45, 3293. (c) Kamal, A.; Kaleem Ahmed, S.; Srinivasa Reddy, K.; Khan, M. N. A.; Shetty, R. V. C. R. N. C.; Siddhardha, B.; Murthy, U. S. N.; Khan, I. A.; Kumar, M.; Sharma, S.; Ram, A. B., Bioorg. Med. Chem. Lett. 2007, 17, 5419.

3. (a) Moldovan, C. M.; Oniga, O.; Pârvu, A.; Tiperciuc, B.; Verite, P.; Pîrnău, A.; Crişan, O.; Bojiţă, M.; Pop, R., Eur. J. Med. Chem. 2011, 46, 526. (b) Kümmerle, A. E.; Vieira, M. M.; Schmitt, M.; Miranda, A. L. P.; Fraga, C. A. M.; Bourguignon, J.-J.; Barreiro, E. J., Bioorg. Med. Chem. Lett. 2009, 19, 4963. (c) Gökçe, M.; Utku, S.; Küpeli, E., Eur. J. Med. Chem. 2009, 44, 3760

4. Leal, C. M.; Pereira, S. L.; Kümmerle, A. E.; Leal, D. M.; Tesch, R.; de Sant'Anna, C. M. R.; Fraga, C. A. M.; Barreiro, E. J.; Sudo, R. T.; Zapata-Sudo, G., Eur. J. Med. Chem. 2012, 55, 49.

5. Fattorusso, C.; Campiani, G.; Kukreja, G.; Persico, M.; Butini, S.; Romano, M. P.; Altarelli, M.; Ros, S.; Brindisi, M.; Savini, L.; Novellino, E.; Nacci, V.; Fattorusso, E.; Parapini, S.; Basilico, N.; Taramelli, D.; Yardley, V.; Croft, S.; Borriello, M.; Gemma, S., J. Med. Chem. 2008, 51, 1333.

6. (a) Liu, T.; Sun, C.; Xing, X.; Jing, L.; Tan, R.; Luo, Y.; Huang, W.; Song, H.; Li, Z.; Zhao, Y., Bioorg. Med. Chem. Lett. 2012, 22, 3122. (b) Despaigne, A. A. R.; Parrilha, G. L.; Izidoro, J. B.; da Costa, P. R.; dos Santos, R. G.; Piro, O. E.; Castellano, E. E.; Rocha, W. R.; Beraldo, H., Eur. J. Med. Chem. 2012, 50, 163.

7. Carvalho, S. A.; Feitosa, L. O.; Soares, M.; Costa, T. E. M. M.; Henriques, M. G.; Salomão, K.; de Castro, S. L.; Kaiser, M.; Brun, R.; Wardell, J. L.; Wardell, S. M. S. V.; Trossini, G. H. G.; Andricopulo, A. D.; da Silva, E. F.; Fraga, C. A. M., Eur. J. Med. Chem. 2012, 54, 512.

8. Cutshall, N. S.; Onrust, R.; Rohde, A.; Gragerov, S.; Hamilton, L.; Harbol, K.; Shen, H.-R.; McKee, S.; Zuta, C.; Gragerova, G.; Florio, V.; Wheeler, T. N.; Gage, J. L., Bioorg. Med. Chem. Lett. 2012, 22, 5595.

9. Wu, A. M.; Senter, P. D., Nat. Biotechnol. 2005, 23, 1137.

10. (a) Yang, M.-H.; Matikonda, S. S.; Altman, R. A., Org. Lett. 2013, 15, 3894. (b) Maruoka, K.; Oishi, M.; Yamamoto, H., J. Am. Chem. Soc. 1996, 118, 2289. (c) Adlington, R. M.; Barrett, A. G. M., Acc. Chem. Res. 1983, 16, 55. (d) Shapiro, R. H.; Lipton, M. F.; Kolonko, K. J.; Buswell, R. L.; Capuano, L. A., Tetrahedron Lett. 1975, 16, 1811.

11. (a) May, J. A.; Stoltz, B. M., J. Am. Chem. Soc. 2002, 124, 12426. (b) Bamford, W. R.; Stevens, T. S., J. Chem. Soc. 1952, 4735.

12. (a) Furrow, M. E.; Myers, A. G., J. Am. Chem. Soc. 2004, 126, 5436. (b) Szmant, H. H.; Harmuth, C. M., J. Am. Chem. Soc. 1964, 86, 2909. (c) Wolff, L., Liebigs Ann. Chem. 1912, 394, 86. (d) Kishner, N., J. Russ. Phys. Chem. Soc. 1911, 43, 582.

13. Deng, X.; Mani, N. S., J. Org. Chem. 2008, 73, 2412.

14. (a) Crisalli, P.; Kool, E. T., J. Org. Chem. 2013, 78, 1184. (b) Dirksen, A.; Dirksen, S.; Hackeng, T. M.; Dawson, P. E., J. Am. Chem. Soc. 2006, 128, 15602. (c) Huc, R. N.; Ivan, Chem. Commun. 2003, 942.

15. Japp, F. R.; Klingemann, F., Ber. Dtsch. Chem. Ges. 1887, 20, 2942.

16. Kozikowski, A. P.; Floyd, W. C., Tetrahedron Lett. 1978, 19, 19

17. Chavannavar, A. P.; Oliver, A. G.; Ashfeld, B. L., Chem. Commun. 2014, 50, 10853.

18. (a) Ghozlan, S. A. S.; Abdelhamid, I. A.; Hatem, G.; Elnagdi, M. H., J. Chem. Res. 2004, 2004, 789. (b) Engel, P. S.; Wang, C.; Chen, Y.; Ruechardt, C.; Beckhaus, H. D., J. Am. Chem. Soc. 1993, 115, 65.

19. Product structure determined by X-ray crystallography. See supporting information for details.

20. Osman, F. H.; El-Samahy, F. A., Chem. Rev. 2002, 102, 629.

21. (a) Ramirez, F., Acc. Chem. Res. 1968, 1, 168. (b) Ramirez, F.; Patwardhan, A. V.; Smith, C. P., J. Org. Chem. 1965, 30, 2575. (c) Ramirez, F.; Desai, N. B., J. Am. Chem. Soc. 1963, 85, 3252.

22. Wei, Y.; Shi, M., Chem. Rev. 2013, 113, 6659. 


\section{Graphical Abstract}

To create your abstract, type over the instructions in the template box below.

Fonts or abstract dimensions should not be changed or altered.

Phosphine-Mediated Addition of 1,2-

Dicarbonyls to Diazenes: An Umpolung Approach toward $N$-Acyl Hydrazone Synthesis

Kasey C. Haugen, Kevin X. Rodriguez, Aditi P. Chavannavar, Allen G. Oliver and Brandon L. Ashfeld ${ }^{*}$

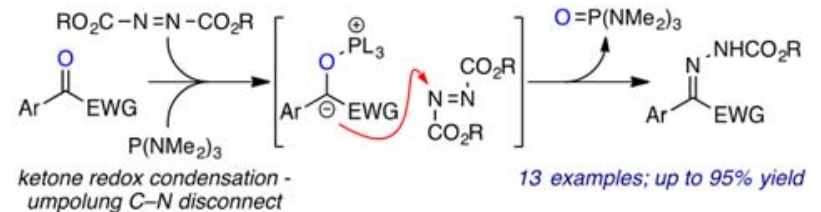

